\title{
Predicting Alzheimer's disease: a polygenic hazard score
}

\author{
AJ Larner ${ }^{1}$, RM Bracewell ${ }^{2}$
}

Title Genetic assessment of age-associated Alzheimer disease risk: development and validation of a polygenic hazard score

\author{
Correspondence to: \\ AJ Larner \\ Cognitive Function Clinic \\ Walton Centre NHS \\ Foundation Trust \\ Liverpool L9 7LJ \\ UK
}

Declaration of interests: RMB is Editor-in-chief of the JRCPE
Email:

a.larner@thewaltoncentre. nhs.uk

\section{Summary}

The genetic factors that influence the development of Alzheimer's disease (AD) have been the subject of large international collaborative studies in recent years involving many thousands of patients and controls. Genome wide association screens (GWAS) have produced large datasets which include not only genetic linkage information but also clinical material such as age of disease onset and, in some cases, in vivo biomarker information (neuroradiological measures of regional brain atrophy; cerebrospinal fluid (CSF) protein markers of AD, Abeta1-42 and tau) and follow-up neuropathological data. These large datasets were used in the current study to integrate genetic information into an epidemiological framework for risk prediction through the construction of a 'polygenic hazard score' (PHS) for lateonset, 'sporadic' AD, the most common form of the disease.

The authors' assessed nearly 2,000 single nucleotide polymorphisms (SNPs) reported to increase the genetic risk of AD in case-control studies of US-based individuals of non-Hispanic European ancestry. Of these, 31 SNPs were included in the final polygenic model - the PHS. The authors also included two variants of the apolipoprotein $\mathrm{E}(\mathrm{ApoE})$ gene, which is known to be the most significant genetic predisposing factor for 'sporadic' AD. By combining an individual's PHS with population-based age-specific incidence rates, the annualised incidence rate (that is, risk of developing $A D$ ) for that individual could be calculated.

To assess the model thus developed, replication studies were undertaken in independent patient samples (replication $n>$ 20,000 individuals). Both the development of the PHS and its validation were done on individuals drawn from specialised memory clinics in the USA. The PHS successfully stratified individuals into different risk strata. Individual genetic profile and age could be translated into incidence rates, with PHSpredicted incidence strongly associated with empirical progression rates. In other words, individual differences in risk of developing $A D$ could be quantified as a function of patient genotype and age. The predicted age of disease onset was strongly associated with the actual age of onset: those with the highest PHS scores developed the disease at around age 84 , while those with the lowest scores were diagnosed around age 95 .

The PHS was also examined with respect to markers of disease, both neuropathology and biomarkers (neuroradiological measures and CSF protein markers). The PHS was significantly associated with standard measures of neurofibrillary tangle (Braak score) and neuritic amyloid plaque pathology (CERAD score); with decreased CSF Abeta1-42 and increased CSF total-tau; and with greater neuroradiological volume loss in the medial temporal lobe (entorhinal cortex and hippocampus).

The authors suggest that the PHS may thus be used as a tool to estimate individual differences in AD risk across the patient's lifetime and to quantify the yearly incidence rate for developing AD.

\section{Opinion}

The high public profile of dementia in general, and of $A D$ in particular, in recent years has unsurprisingly caused numbers of older (and not so old) people to worry that they might be developing or going to develop AD. Concurrently, the failure of many $A D$ treatment trials in recent years has

${ }^{1}$ Consultant Neurologist, Walton Centre NHS Foundation Trust, Liverpool, UK; ${ }^{2}$ Consultant Neurologist, Walton Centre NHS Foundation Trust, Liverpool, UK; Consultant Neurologist, Betsi Cadwaladr University Health Board, North Wales; and Senior Lecturer in Behavioural Neurology and Cognitive Neuroscience, Bangor University, Bangor, UK 
enhanced the view that disease prevention, rather than 'cure' following diagnosis, is the most productive route forward, assuming that some form of disease modifying therapy will be discovered in the future.

Hitherto, no accurate, individually tailored, prediction of $A D$ diagnosis could be made, save in those relatively rare individuals with a family history showing that $A D$ was inherited as an autosomal dominant condition, and where the pathogenic mutation was defined in one or more affected family members, in one of the three genes in which mutations are known to be deterministic for $A D$, namely amyloid precursor protein (APP), and presenilin 1 and 2 (PSEN1, PSEN2). Mutations in PSEN1, the most common recognised cause of autosomal dominant $A D$, have essentially $100 \%$ penetrance. These cases are almost invariably associated with the unusual early-onset form of AD. ${ }^{1}$

In addition to deterministic mutations, a number of genetic predisposing factors, of themselves neither necessary nor sufficient to cause AD, have been identified. Of these, the best known relates to ApoE genotype. The use of GWAS has broadened the number of identified possible genetic risk factors. In general, individual SNPs identified in GWAS raise the risk of disease only slightly, but together may significantly affect the risk of developing a disease.

Risk scores for prediction of dementia have been previously constructed, based on recognised mid-life risk factors such as hypertension and hypercholesterolaemia. ${ }^{2}$ However, the integration of genetic information with epidemiological background offers a far more sophisticated and potent method of risk prediction.

Potential uses of the PHS include the enrichment of patient cohorts, based on stratified risk, in prevention and therapeutic trials (previous clinical trials may have failed, at least in part, because of inclusion of age-matched controls who were at high risk of progression to disease). Whether the 'genomic profile' might be used by individual patients for the purpose of future planning is an intriguing, but hitherto unexplored, possibility. Use of the PHS as a possible screening test at a population level might also be possible, if fraught with ethical issues. In this regard, an important caveat to the current study is its focus on patients of European descent. Replication in non-white populations, and prospective validation of the PHS in community-based cohorts, is required.
The approach used in this study is illustrative of an emerging trend, namely the development of 'bioprediction' of brain disorder. This represents a reorientation of the medical concept of 'disorder' which rejects the old binary or categorical formulation (disorder/normalcy) in favour of a probabilistic model based on present and future risks of harm. Such an approach is justified in part by the belief that disease biomarkers will not map cleanly onto clinical diagnostic categories. Baum has explored the bioethical issues, and has proposed a 'probability dysfunction' model in which disorders are conceptualised as graphs of probability over time, the area under which would help to separate out self-limiting disorders from those with low probabilities of harm over longer time periods. 'Risk banding', based on the shape of the probability function, is the strategy advocated to determine the necessity or otherwise for response/ intervention. ${ }^{3}$ The PHS may be seen as a probability function which might be used to address risk of AD. I

\section{References}

1 Larner AJ, Doran M. Clinical phenotypic heterogeneity of Alzheimer's disease associated with mutations of the presenilin-1 gene. J Neurol 2006; 253: 139-58.

2 Kivipelto M, Ngandu T, Laatikainen T et al. Risk score for the prediction of dementia in 20 years among middle aged people: a longitudinal, population-based study. Lancet Neurol 2006; 5: 735-41.

3 Baum ML. The Neuroethics of Biomarkers: What the Development of Bioprediction Means for Moral Responsibility, Justice, and the Nature of Mental Disorder. Oxford: Oxford University Press; 2016. 